\title{
Spondylarthrite ankylosante et atteinte de l'articulation temporo-mandibulaire : à propos d'un cas
}

\author{
Lerici S, Fricain JC \\ UFR d'Odontologie, Bordeaux, France \\ seblerici@gmail.com
}

La spondylarthrite ankylosante est une maladie rhumatismale inflammatoire chronique touchant préférentiellement les structures axiales, rachis et os iliaque. Les articulations périphériques peuvent théoriquement être atteintes. Les cas publiés de manifestations intéressant les articulations temporo-mandibulaires (ATM) sont plus rares (Helenius et al. 2006, Manemi et al. 2009)

Le cas rapporté correspond à celui d'une femme de 61ans, adressée pour avulsions multiples. Elle présentait à l'examen des télangiectasies en rapport avec une maladie de Rendu-OslerWeber. Elle souffrait également d'une spondylarthrite ankylosante ; il n'y a pas de liens connus entre cette affection et la maladie de Rendu-Osler-Weber. L'inspection et la palpation de l'ATM ont révélé des bruits articulaires associés à une douleur à la palpation temporale droite, ainsi qu'une diminution de l'ouverture buccale.

Une radiographie de l'ATM a été demandée pour évaluer l'intégrité condylienne en position bouche fermée, et sa mobilité en ouverture. Le but était de mettre en corrélation l'atteinte articulaire avec la pathologie de la patiente, afin de proposer une prise en charge adaptée.

Cet examen a mis en évidence une nette diminution de l'amplitude du mouvement du condyle gauche par rapport au condyle droit, pouvant faire suspecter un début d'ankylose. En effet, les signes radiographiques retrouvés dans la littérature (Miia 2005) comportent une superposition du condyle avec l'éminence temporale, aspect qui est retrouvé chez cette patiente. Il s'agit donc d'une atteinte précoce de l'ATM avec une nette diminution des mouvements articulaires, dûe à une altération des surfaces articulaires et à une disparition de l'interligne articulaire.

Le traitement d'une ankylose de l'ATM, qu'elle soit fibreuse ou osseuse, est essentiellement chirurgical (libération chirurgicale du condyle, interposition d'un lambeau musculaire ou mise en place d'une prothèse condylienne) (Vasconcelos et al. 2009). Une prise en charge précoce permettrait d'améliorer l'amplitude articulaire par des exercices de kinésithérapie et de retarder la nécessité d'une intervention chirurgicale.

La spondylarthrite ankylosante n'entraîne pas de prise en charge bucco-dentaire particulière ; toutefois, un examen systématique et attentif de l'ATM (radiographie, tomodensitométrie ou imagerie par résonance magnétique) chez ces patients est nécessaire afin de diagnostiquer précocement une ankylose et d'en limiter l’aggravation par une prise en charge adaptée. 